\title{
Successful Treatment of Refractory Cardiogenic Shock and Electrical Storm Using the IMPELLA 5.0 with Atrial Overdrive Pacing, in a Patient with Severe Peripheral Arterial Disease
}

\author{
Tomoya Hasegawa, ${ }^{1}$ MD, Takahiro Tachibana, ${ }^{1}$ MD, Hikaru Kimura, ${ }^{1}$ MD and Yoshikazu Yazaki, ${ }^{1} \mathrm{PhD}$
}

\begin{abstract}
Summary
Cardiogenic shock with electrical storm is a challenging condition to manage in patients with acute myocardial infarction despite primary percutaneous coronary interventions. While active mechanical circulatory support devices may improve hemodynamics in this situation, identifying the appropriate arterial access for device deployment is difficult in patients with severe peripheral arterial disease due to severe stenosis or obstruction and tortuous path of the femoral-iliac artery or descending aorta; additionally, this also reduces the mechanical viability of the implanted circulatory support devices, thus posing a risk for limb ischemia. Herein, we report on the effectiveness of the IMPELLA 5.0, deployed via the axillary artery, in combination with atrial overdrive pacing to manage a patient with cardiogenic shock and electrical storm, without extracorporeal membrane oxygenation. Our strategy, which does not require access via the groin area, may be an attractive option for patients with severe peripheral arterial disease, particularly those with aorto-iliac occlusive disease.
\end{abstract}

(Int Heart J 2021; 62: 677-681)

Key words: Mechanical circulatory support device

$\mathrm{C}$ ardiogenic shock (CS) secondary to acute myocardial infarction (AMI) remains a medically challenging event to manage, with a high mortality rate despite aggressive revascularization to non-culprit lesions and intra-aortic balloon pumping (IABP). ${ }^{1,2)} \mathrm{A}$ recent study indicated that although the therapeutic effectiveness of active mechanical circulatory support (MCS) devices has been proven based on a limited body of evidence, there is still good evidence that the IMPELLA may provide better hemodynamic control ${ }^{3)}$ and survival rate ${ }^{4)}$ than IABP. Unfortunately, severe peripheral artery disease (PAD) leads to poor access to a vascular site required for MCS devices, including the IMPELLA, resulting in a poor prognostic outcome of CS. Herein, we present a case that highlights the effectiveness of the IMPELLA 5.0, deployed via the axillary artery, combined with temporary atrial overdrive pacing, to stabilize hemodynamics in a patient with PAD during refractory $\mathrm{CS}$ and electrical storm (ES).

\section{Case Report}

A 66-year-old man (weight: $68 \mathrm{~kg}$, body mass index: 23.6) presented to our emergency department with a sudden onset of chest pain and orthopnea. He had undergone endovascular repair of an abdominal aortic aneurysm.
During the subacute phase, he developed an acute occlusion in the right limb of the endograft (Figure 1), which required an emergent left-to-right femorofemoral bypass. Other relevant history included dyslipidemia, a family history (father) of myocardial infarction, and habitual smoking. On admission, the patient was hemodynamically unstable, with a blood pressure of $114 / 91 \mathrm{~mm} \mathrm{Hg}$, a pulse rate of 150 beats/min, and frequent non-sustained ventricular tachycardia. He developed tachypnea, with an oxygen saturation of $92 \%$ on a reservoir face mask (10 L). A 12-lead electrocardiogram (ECG) and echocardiography revealed a broad anterior ST-elevation myocardial infarction (STEMI). Chest radiographs showed severe pulmonary congestion, Killip class III.

Emergent coronary angiography, performed via the radial artery, revealed a subtotal occlusion in the mid-left anterior descending artery (LAD) and the distal right coronary artery (RCA), with a $90 \%$ stenosis of the midleft circumflex artery (LCX) (Figure 1B). We proceeded with IABP and temporary right ventricular (60/minute) pacing via left femoral access and intubated the patient to manage this hemodynamic instability and respiratory insufficiency. Two drug-eluting stents (DESs) were deployed in the LAD. A final angiography revealed thrombolysis in myocardial infarction (TIMI) grade 3 flow. After the primary percutaneous cardiac intervention (PCI), the patient's

From the ${ }^{1}$ Division of Cardiology, Saku Central Hospital Advanced Care Center, Nagano, Japan.

Address for correspondence: Tomoya Hasegawa, MD, Division of Cardiology, Saku Central Hospital Advanced Care Center, $3400-28$ Nakagomi, Saku, Nagano 385-0051, Japan. E-mail: tomoya.hasegawa0825@gmail.com

Received for publication July 10, 2020. Revised and accepted December 7, 2020.

Released in advance online on J-STAGE May 15, 2021.

doi: 10.1536/ihj.20-487

All rights reserved by the International Heart Journal Association. 

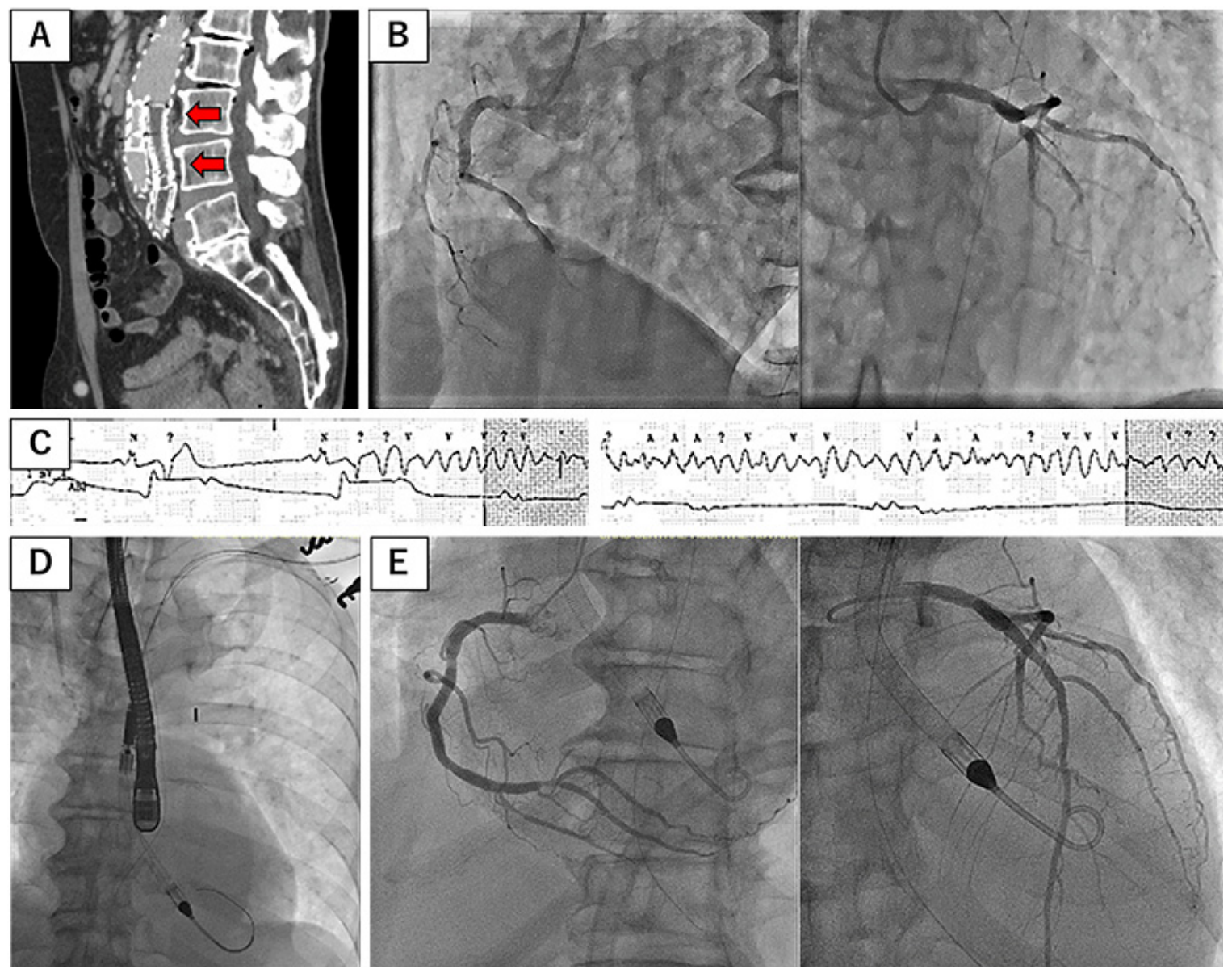

Figure 1. A: Contrast-enhanced computed tomography (CT), obtained in the subacute phase after endovascular repair of the abdominal aneurysm, showing a thrombotic occlusion of the right endograft limb (red arrow). B: Pre-interventional images, with coronary angiography revealing a subtotal occlusion of the mid-portion of the left anterior descending artery (LAD) and the distal right coronary artery (RCA), a chronic total occlusion in the first obtuse marginal branch, and 90\% stenosis in the mid-left circumflex artery (LCX). C: Electrocardiogram showing the onset of an electrical storm ( $\mathrm{R}$ on $\mathrm{T}$ phenome). D: Peri-procedural image showing the IMPELLA 5.0 device surgically implanted via the left subclavian artery. E: Repeated coronary angiography demonstrating the patency of the LAD treated using two overlapping drug-eluting stents (DESs), of LCX treated with short DES, and of RCA treated with tandem DESs, respectively.

blood pressure was $84 / 63 \mathrm{~mm} \mathrm{Hg}$, the pulse rate was 93 beats/min, and his oxygen saturation was $92 \%\left(\mathrm{FiO}_{2} 0.5\right)$. A norepinephrine infusion of $>0.2 \mathrm{mcg} / \mathrm{kg} / \mathrm{minute}$ was required to support his cardiac status.

Despite revascularization to the LAD vessel, hypotension and oliguria persisted despite the increasing vasopressor doses and diuretic infusion. The patient was diagnosed with acute CS, and the IABP-resistant residual ischemia was suspected to cause the deterioration. Complete revascularization to the other coronary arteries was attempted at 24 hours post-admission, with good restoration of coronary flow, as observed on coronary angiography obtained after deployment of multiple DESs to the RCA and LCX. However, although we succeeded in achieving hemodynamic stability (blood pressure, 115/58 $\mathrm{mm} \mathrm{Hg}$; pulse rate, 58 beats/minute; and oxygen saturation, $\left.95 \%\left(\mathrm{FiO}_{2} \mathrm{0.3}\right)\right)$ after additional coronary interventions, on the next day, the patient presented several episodes of ventricular fibrillation (VF), which degenerated into ES (Figure 1C).
The patient was resistant to efficacious resuscitation strategies, including amiodarone loading and deeper sedation therapy. Coronary circulation was spontaneously restored with repeated electrical defibrillation. During this episode, there were no electrolyte abnormalities and no evidence of ST-segment elevation on the 12-lead ECG, and the QT interval was normal. Echocardiography revealed that left ventricular function was severely impaired, with pulmonary edema visible on chest radiographs and elevated serum lactate levels on arterial blood gas analysis. These findings indicated the need for more solid MCS devices, including extracorporeal membrane oxygenation (ECMO), to re-stabilize the patient.

No viable access sites were available for MCS implantation as the right femoral artery was occluded and the left femoral artery was occupied by the IABP. Moreover, the patient's cardiac status did not allow for conversion from IABP to another higher-level MCS device. After consultation with the cardiac team, an IMPELLA 5.0 was successfully inserted via a left axillary surgical approach 

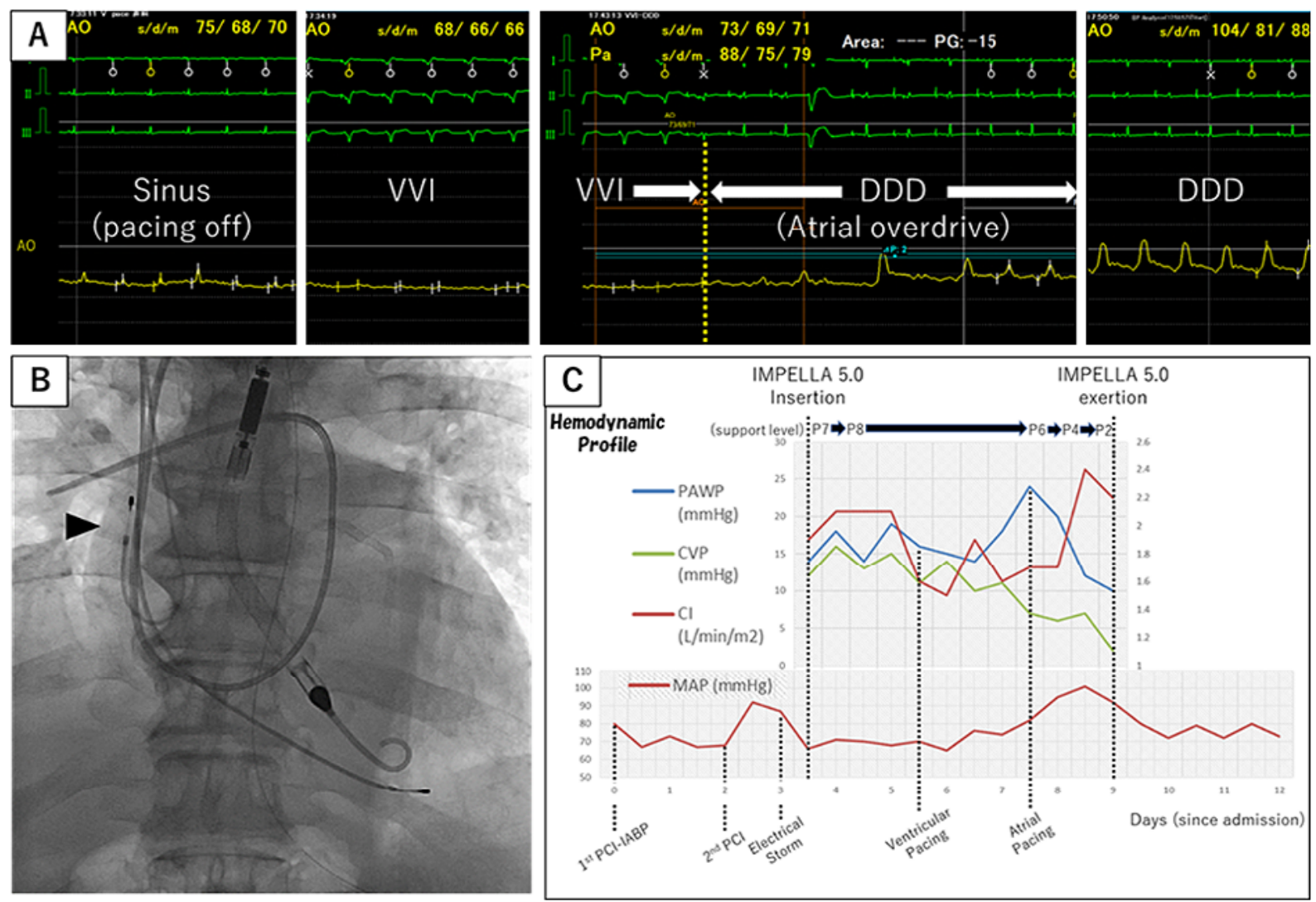

Figure 2. A: Peri-procedural of IMPELLA electrocardiogram and arterial pressure waveform (APW) images following IMPELLA 5.0 implantation. In the sinus rhythm (pacing off) (1), the mean arterial pressure (MAP) is almost $70 \mathrm{mmHg}$ with low pulse pressure and a narrow QRS wave. In the ventricular pacing phase (2), the QRS wave is widened, and the systolic blood pressure (SBP) decreased, resulting in a worsening of the MAP, with the APW image appearing almost flat. In the arterial pacing phase (3), the QRS wave narrows, and the SBP is increased. Finally, the MAP reaches almost $90 \mathrm{mmHg}$. B: The additional temporary atrial pacing catheter is inserted with an active fixation catheter (black arrowhead). C: This illustration shows the hemodynamic profiles and MAP during intensive care unit stay. Pulmonary artery wedge pressure, cardiac index, and MAP significantly improved after the onset of atrial overdrive pacing. PAWP indicates pulmonary artery wedge pressure; CVP, central venous pressure; $\mathrm{CI}$, cardiac index; and MAP, mean arterial pressure.

(Figure 1D), followed by removing the IABP. Temporary ventricular pacing was maintained until confirmation of the patency of implanted stents on repeated coronary angiography (Figure 1E). The ventricular pacing was discontinued as its stimulant effect induced a premature ectopic beat resulting in malignant arrhythmia. Within a few hours after implantation of the IMPELLA 5.0, the patient's hemodynamic parameters improved, with a decrease in serum lactate levels and spontaneous recovery of diuresis. A chest radiograph confirmed a decrease in pulmonary congestion. However, the malignant ventricular arrhythmia persisted. We did consider the possibility of re-implanting overdrive ventricular pacing to avoid the need for repeated electrical defibrillation; however, due to the severity of the ventricular dysfunction, ventricular pacing may have had deleterious effects. The right ventricular pacing (90/minute) at the time of implantation of the IMPELLA 5.0 caused an increase in the pulmonary artery wedge pressure (PAWP), with a worsening of the cardiac index (CI) and a decrease in systolic blood pressure (Figure 2A, C). An additional inotrope agent would not have resolved the situation. To address the issue, we added temporary atrial overdrive pacing to suppress the ventricle stress (Figure 2 B). This initial atrial pacing (90/minute), which led to intrinsic atrioventricular conduction, narrowed the QRS wave, induced an appropriate PAWP, and increased the MAP (Figure 2A, C). The mechanical support by IMPELLA 5.0 and atrial overdrive pacing allowed us to optimize his medication, including the beta-blockers (bisoprolol). Left ventricular contraction gradually improved, with pulmonary edema resolution and the odious malignant arrhythmia within a few days. The patient was successfully extubated 4 days after implanting the IMPELLA 5.0, followed by careful weaning from the MCS device and inotrope/vasopressor. The IMPELLA 5.0 was surgically removed 6 days after implantation. Although the patient's medical course was complicated by paroxysmal atrial fibrillation and a small hemorrhagic cerebral infarction of the left occipital lobe during his stay in intensive care, he remained well and without remarkable neurological deficits. Twenty-eight days after the index admission, a transvenous implantable cardiac defibrillator was implanted. Daily medications at discharge were as follows: prasugrel $3.75 \mathrm{mg}$, edoxaban $30 \mathrm{mg}$, enalapril $2.5 \mathrm{mg}$, bi- 
soprolol $5 \mathrm{mg}$, amiodarone $100 \mathrm{mg}$, tolvaptan $3.75 \mathrm{mg}$, furosemide $10 \mathrm{mg}$, atorvastatin $20 \mathrm{mg}$, and lansoprazole $15 \mathrm{mg}$. Within 6 months after discharge, there was no incidence of fatal arrhythmia or ischemic events.

\section{Discussion}

Our case highlights the effectiveness of IMPELLA 5.0 and atrial overdrive pacing for a patient with CS and ES refractory to hemodynamic support with IABP. Since severe PAD coexisted in this case, we inserted the IMPELLA 5.0 via an axillary approach without involving the groin.

Moreover, left ventricular unloading produced by the IMPELLA 5.0 and the physiologically well-suited atrial pacing may have promoted the recovery from ischemic myocardial stunning. To the best of our knowledge, this is the first case reporting a successful treatment combining IMPELLA and atrial overdrive pacing for a patient with CS and ES due to acute myocardial infarction.

IMPELLA 5.0 support for a patient with severe PAD patient with CS and ES: CS and ES remain challenging, and often fatal, complications of STEMI, despite optimal medical therapy and complete revascularization. ${ }^{1)}$ For the past few decades, IABP has been performed to manage refractory $\mathrm{CS}$, even with the lack of evidence from highquality clinical trials. ${ }^{5)}$ Venoarterial ECMO remains the fastest deployable device to stabilize a patient in acute CS resistant to IABP support. However, ECMO has poor outcomes on $\mathrm{MI}{ }^{6}{ }^{6}$ as it cannot reduce the load on the left ventricle and causes an extremely high afterload. These factors worsen coronary perfusion and increase ischemic damage to the myocardium. Moreover, ECMO is associated with an inherent risk of lower limb ischemia. Although an active MCS device is helpful, viable access for its implantation is not always possible in patients with severe PAD. The American Association for Thoracic Surgery/International Society for Heart and Lung Transplantation guidelines state that extensive atherosclerotic disease may preclude candidacy for LVAD support. ${ }^{7)}$ This underlines the need for a simple system that can be used to implant from a site other than the groin while still providing aggressive support for left ventricular unloading - all of which are possible with IMPELLA 5.0 and with an option to deploy via an axillary approach. ${ }^{6}$

Advantages of IMPELLA 5.0 compared with other IMPELLA series for myocardial damage: A randomized trial of CS among patients with an AMI indicated that the IMPELLA improved the hemodynamic profile $\mathrm{e}^{3)}$ and decreased lactate levels ${ }^{8)}$ compared to IABP. The IMPELLA can restore tissue perfusion, reduce the work of the left ventricle, and assist in recovery from myocardial stunning. Importantly, it also provides additional support provided for residual ischemia/infarcted myocardial damage. In their animal model, Saku, et al. demonstrated that the total support provided by the IMPELLA 5.0 markedly reduces the left ventricular work and the infarct size to a greater extent than the partial support provided by the IMPELLA 2.5.9) The additional support provided by the IMPELLA 5.0 may prevent left ventricular remodeling and subsequent heart failure.9) We note that although the IM-
PELLA CP can provide sufficient support to reduce the left ventricular loading, our institution could not use the IMPELLA CP at the time the case was presented. Considering that our patient was a well-built man, we consider that our selection of the IMPELLA 5.0 was appropriate to produce the total support needed. It is important to note that the IMPELLA supports only left ventricular performance, which is a distinct disadvantage. If the right heart failure remains complicated after IMPELLA support, a right ventricular support device, such as venoarterial ECMO or the IMPELLA RP, should be inserted to provide dual support, although the IMPELLA RP is not available in Japan. In the present case, we were able to stabilize the patient's status before the emergence of several VF episodes with additional revascularization of residual areas of ischemia. There were no signs of right ventricular failure during these procedures. Therefore, in our opinion, only a strong support for the left ventricle load was required for our patient. Accordingly, the IMPELLA 5.0 was effective in our case, producing powerful, independent, ventricular support (via a single site approach), did not provoke lower extremity ischemia, and enabled a more stable positioning of the catheter than a groin placement. These advantages improved myocardial conditions, as well as supporting early extubation and rehabilitation. Based on our experience, we consider the IMPELLA 5.0, surgically implanted via the axillary artery, as the best option to stabilize CS with ES among patients with severe PAD, limiting viable access for circulatory device implantation.

Atrial overdrive pacing under the IMPELLA 5.0 support for a patient suffering from ES: However, the use of the IMPELLA alone may not be an appropriate option for the management of drug-resistant ES. In these cases, ECMO should generally remain the first option. However, if additional temporary overdrive pacing can be provided before complete circulatory collapse, our combination strategy with the IMPELLA may provide a simple modality to control ventricular arrhythmia. In the recent European Society of Cardiology guidelines, transvenous catheter overdrive stimulation is recommended to treat recurrent ventricular arrhythmia resistant to drug therapy. ${ }^{10}$ However, it is important to be mindful that ventricular overdrive pacing in patients with severe impairment in left ventricular function due to AMI may induce another malignant arrhythmia. By comparison, atrial overdrive pacing was an effective and safe method for treating our patient who had severe left ventricular dysfunction. ${ }^{11)}$ Atrial overdrive pacing provided intrinsic physiological stimulation, which narrowed the QRS complex and promoted increased cardiac output while avoiding the harmful effect of ventricular pacing. Therefore, in our case, using the IMPELLA 5.0 device in combination with atrial overdrive pacing dynamically improved our patient's hemodynamic profile and MAP (Figure 2C). This low-stressed physiological stimulation and the capacity for up-titration to the optimal dose of beta-blocker due to atrial overdrive pacing contributed to the attenuation of ES.

Important caution about our strategy of the IMPELLA and atrial overdrive pacing: We note that our use of the IMPELLA and atrial overdrive pacing would be 
limited to treating patients with CS and/or ES. The importance of our case report is particularly with respect to the support for CS; hence, the risk of mortality in these patients is extremely high, although evidence regarding the effectiveness of the IMPELLA to improve clinical outcomes is currently limited. ${ }^{12)}$ Thus, consideration for escalating care to a higher level of MCS, such as an increase from only the IMPELLA to additional venoarterial ECMO (Management of ECPELLA), is required whenever these patients worsen. Charlene, et al. ${ }^{13)}$ recommended that a higher level MCS should be considered for patients with a higher lactate level $(>6.8 \mathrm{mmol} / \mathrm{L})$, lower $\mathrm{pH}(<7.24)$, lower serum $\mathrm{CO}_{2}(<19.1 \mathrm{mmol} / \mathrm{L})$, and/or requiring $\geq 5$ vasopressors and inotropes. Retrospectively, none of these criteria were fulfilled in our case. Similarly, as mention above, our strategy may not be indicated in on-going circulatory collapse, such as refractory ES. Nonetheless, we propose that the use of the IMPELLA in combination with atrial overdrive pacing would be suitable for patients in CS and/or ES, except in cases of extreme hemodynamic instability.

\section{Conclusion}

In conclusion, the use of the IMPELLA 5.0 in combination with atrial overdrive pacing may be an effective strategy against refractory ES for patients with CS resulting from an AMI. In particular, prompt axillary arterial implantation of an IMPELLA 5.0 without the need for a groin approach may provide an attractive option for patients with severe PAD.

\section{Disclosure}

\section{Conflicts of interest: None.}

\section{References}

1. Thiele H, Akin I, Sandri M, et al. CULPRIT-SHOCK Investigators. One-year outcome after PCI strategies in cardiogenic shock. N Engl J Med 2018; 379: 1699-710.

2. Thiele H, Zeymer U, Neumann FJ, et al. Intra-aortic balloon support for myocardial infarction with cardiogenic shock. N Engl J Med 2012; 367: 1287-96.

3. Seyfarth M, Sibbing D, Bauer I, et al. A randomized clinical trial to evaluate the safety and efficacy of a percutaneous left ventricular assist device versus intra-aortic balloon pumping for treatment of cardiogenic shock caused by myocardial infarction. J Am Coll Cardiol 2008; 52: 1584-8.

4. O'Neill WW, Schreiber T, Wohns DW, et al. The current use of Impella 2.5 in acute myocardial infarction complicated by cardiogenic shock: results from the USpella Registry. J Interv Cardiol 2014; 27: 1-11.

5. Thiele H, Zeymer U, Neumann FJ, et al. Intra-aortic balloon counterpulsation in acute myocardial infarction complicated by cardiogenic shock. Final 12-month results of the randomised Intra-aortic Balloon Pump in Cardiogenic Shock II (IABPSHOCK II) Trial. Lancet 2013; 382: 1638-45.

6. Shishehbor MH, Moazami N, Tong MY, et al. Cardiogenic shock: From ECMO to Impella and beyond. Cleve Clin J Med 2017; 84: 287-95

7. Kirklin JK, Pagani FD, Goldstein DJ, et al. American Association for Thoracic Surgery/International Society for Heart and Lung Transplantation guidelines on selected topics in mechanical circulatory support. J Heart Lung Transplant 2020; 39: 187219.

8. Ouweneel DM, Eriksen E, Sjauw KD, et al. Impella CP versus intra-aortic balloon pump support in acute myocardial infarction complicated by cardiogenic shock. The IMPRESS in Severe Shock trial. J Am Coll Card 2017; 69: 278-87.

9. Saku K, Kakino T, Arimura T, et al. Left ventricular mechanical unloading by total support of Impella in myocardial infarction reduces infarct size, preserves left ventricular function and prevents subsequent heart failure in dogs. Circ Heart Fail 2018; 11: e004397.

10. Priori SG, Blomström-Lundqvist C, Mazzanti A, et al. 2015 ESC Guidelines for the management of patients with ventricular arrhythmias and the prevention of sudden cardiac death: the Task Force for the Management of Patients with Ventricular Arrhythmias and the Prevention of Sudden Cardiac Death of the European Society of Cardiology (ESC) Endorsed by: Association for European Paediatric and Congenital Cardiology (AEPC). Eur Heart J 2015; 36: 2793-867.

11. Aguiar Rosa S, Oliveira M, Valente B, Silva Cunha P, Almeida Morais L, Cruz Ferreira R. Ventricular electrical storm after acute myocardial infarction successfully treated with temporary atrial overdrive pacing. Med Intensiva 2017; 41: 252-4.

12. Thiele H, Jobs A, Ouweneel DM, et al. Percutaneous short-term active mechanical support devices in cardiogenic shock: a systematic review and collaborative meta-analysis of randomized trials. Eur Heart J 2017; 38: 3523-31.

13. Rohm CL, Gadidov B, Leitson M, Ray HE, Prasad R. Predictors of mortality and outcomes of acute severe cardiogenic shock treated with the Impella device. Am J Cardiol 2019; 124: 499504. 